\title{
Beliefs held by breast surgeons that impact the treatment decision process for advanced breast cancer patients: a qualitative study
}

This article was published in the following Dove Press journal: Breast Cancer: Targets and Therapy

\author{
Reina Ozeki-Hayashi \\ Misao Fujita ${ }^{2}$ \\ Atsushi Tsuchiya ${ }^{3}$ \\ Taichi Hatta ${ }^{2}$ \\ Eisuke Nakazawa' \\ Yoshiyuki Takimoto' \\ Akira Akabayashi ${ }^{1,4}$
}

'Department of Biomedical Ethics, The University of Tokyo Faculty of Medicine, Tokyo, Japan; ${ }^{2}$ Uehiro Research Division for iPS Cell Ethics, Center for iPS Cell Research and Application Kyoto University, Kyoto, Japan; ${ }^{3}$ Industrial and Social Science, Tokushima University Graduate School of Technology,

Tokushima, Japan; ${ }^{4}$ Division of Medical Ethics, Department of Population Health, New York University School of Medicine, New York, NY, USA
Correspondence: Reina Ozeki-Hayashi Department of Biomedical Ethics, University of Tokyo Faculty of

Medicine, 7-3-I Hongo, Bunkyo-ku,

Tokyo II3-0033, Japan

Tel +8I $3584 \mid 35$ II

$\mathrm{Fax}+81358413319$

Email reinaozk-tky@umin.ac.jp
Introduction: Although guidelines do not recommend chemotherapy for patients with advanced cancer when death is imminent, many reports suggest the tendency to continue this treatment has been increasing every year. This study aimed to construct a model to clarify the beliefs and communication of doctors who administer chemotherapy to patients with recurrent or metastatic (hereafter, "recurrent/metastatic") breast cancer, and determine how these beliefs are related to the process of treating patients.

Materials and methods: Semi-structured interviews were conducted with 21 breast surgeons, and interview contents were analyzed using the grounded theory approach in order to conceptualize the treatment process.

Results: The process of chemotherapy for patients with recurrent/metastatic breast cancer differed based on two beliefs held by doctors. One was a "belief that the patient is an entity who cannot accept death," and throughout the treatment process, these doctors consistently avoided sharing bad news that might hurt patients, and always discussed aggressive chemotherapy. They proposed treatments as long as options remained, and when they ultimately judged that the physical condition of patients could not withstand further treatment, treatment was terminated despite the patient hoping for continuation. The other was a "belief that the patient is an entity who can accept death." From early on after recurrence/metastasis, these doctors repeatedly gave patients information including bad news about prognosis, and when they judged that further treatment would hinder a patient's ability to have a good death, they proposed terminating treatment.

Conclusion: We demonstrated that breast surgeons treating recurrent/metastatic breast cancer patients have two beliefs and constructed a model of the treatment process based on those beliefs. This offered breast surgeons, who make decisions regarding treatment without clearly-defined guidelines, a chance to reflect on their own care style, which we believe will contribute to optimal patient care.

Keywords: palliative chemotherapy, end-of-life, decision-making process, qualitative research

\section{Introduction}

Although guidelines of the American Society of Clinical Oncology do not recommend chemotherapy when patients are near death, chemotherapy is continued in some cases until immediately before death. Numerous reports suggest that this trend is increasing every year. ${ }^{1-3}$ Several studies have reported that these decisions to continue chemotherapy are influenced by the beliefs held by doctors and their background, ${ }^{4}$ as well as by a "never give up" relationship fostered between doctors 
and patients. ${ }^{5}$ While some studies have touched on patterns of the treatment process and terminal stage continuation or suspension of chemotherapy, ${ }^{6}$ the manner in which these patterns are influenced by the physician's thinking has not been addressed.

Against this backdrop, the present study examined breast surgeons who perform palliative chemotherapy for patients with recurrent or metastatic (hereafter, "recurrent/ metastatic") breast cancer, aiming to clarify their beliefs, how these beliefs relate to patient-doctor communication, and the treatment process leading from a diagnosis of recurrence/metastasis to patient death. The aim was to construct a model of relationships between the beliefs held by doctors, the palliative chemotherapy process, and patient-doctor communication.

The first reason we narrowed our focus to breast cancer is that breast cancer is highly sensitive to chemotherapy, and the disease appears in patients at a relatively young age. Because these characteristics have been identified as factors associated with doctors continuing chemotherapy into the terminal stage, ${ }^{3}$ we believed it to be highly likely that breast surgeons would propose aggressive anti-cancer therapy for these patients. The second reason is the variety of anti-cancer therapies from which to choose when treating patients with breast cancer recurrence/metastasis. Thus, compared to other cancers, if one treatment is ineffective, other options to continue treatment exist.

\section{Methods}

\section{Participants}

Participants were board certified breast specialists with a track record of treating breast cancer with chemotherapy in Japan. In Japan, doctors who specialize in this area tend to be surgeons rather than oncologists, and perform diagnosis, surgery, and chemotherapy. In selecting participants, we performed theoretical sampling combined with snowball sampling and, in order to collect data in as wide a range as possible (in terms of affiliated hospital size, record of treating patients, personal history and gender, and access to resources in palliative medicine). All participants gave their written informed consent for participate in this study. This study was approved by the research ethics committee of the University of Tokyo Faculty of Medicine (No. 10,317).

\section{Data collection}

All interviews were conducted by one of the authors $(\mathrm{ROH}) . \mathrm{ROH}$ is a physician and has experience working in a department of palliative medicine, and holds a $\mathrm{PhD}$ in qualitative research, and thus is well versed in qualitative studies. In terms of participant background (Table 1), there were 16 men and 5 women, with a median age of 48 years (range, 35-56 years). Semi-structured interviews were conducted in a private room to ensure privacy, for an average of 86 mins (range, 70-120 mins).

An interview guide was created based on previous research on diverse cancer patients as well as pilot interviews to breast surgeons. Participants were questioned about their thoughts and experiences about their practices of deciding chemotherapy for breast cancer recurrence/metastasis. During a later phase of the study, the interview guide was revised to include questions about communication with patients and family members regarding prognosis (Box 1).

\section{Data analysis}

All interviews were audio taped and transcribed verbatim. The process of data collection and analysis followed the Grounded Theory Approach. ${ }^{7}$ Verbatim records of interviews were analyzed using ATLAS.ti.7.1.7 (Science Software Development GmbH, Berlin). Open Coding, Axial Coding, and Selective Coding were performed. Concepts were generated to explain the decision-making process of doctors regarding chemotherapy, and additional data were collected to develop these concepts. Data collection and analysis were continued until theoretical saturation was reached. ${ }^{7}$ To ensure credibility of the analytical process and analytical results, periodic peer debriefings were held with colleagues who conduct qualitative research. ${ }^{8}$ In addition, member checks ${ }^{9}$ were conducted, ie, the authors explained the preliminary analysis report to two participants for feedback.

\section{Results}

\section{Two beliefs about accepting death}

The process by which the chemotherapy of patients with recurrent/metastatic breast cancer was continued and terminated differed based on two beliefs held by doctors (Figure 1). One of these is a "belief that the patient is an entity who cannot accept death" (hereafter "non-acceptance belief"). Doctors with the non-acceptance belief maintained that bad news could harm patients, focused first only on discussions of anti-cancer therapy, and avoided any discussions that might suggest death. They proposed treatments as long as options were available, and ultimately when it was determined that the patient's physical condition could not bear further treatment, 
Table I Doctor background

\begin{tabular}{|c|c|c|c|c|c|c|}
\hline ID & Age & $\begin{array}{l}\text { Years } \\
\text { of experience }\end{array}$ & Sex & Specialist $^{\mathrm{a}}$ & Palliative care ${ }^{b}$ & Hospital function ${ }^{c}$ \\
\hline I & 40 's & 15 & $M$ & Spec. & PCT/PCU & Cancer \\
\hline 2 & 40 's & 19 & $M$ & Spec./Onco & РCT & University \\
\hline 3 & 30 's & 9 & $M$ & Cert. & $\mathrm{PCT} / \mathrm{PCU}$ & Cancer \\
\hline 4 & 40's & 24 & M & Spec. & No & General \\
\hline 5 & 40 's & 17 & $M$ & Spec. & PCT/PCU & Cancer \\
\hline 6 & 50 's & 27 & $\mathrm{~F}$ & Spec. & РCT & General \\
\hline 7 & 50 's & 27 & $M$ & Spec. & PCT & General \\
\hline 8 & 50 's & 30 & $F$ & Spec. & PCT & General \\
\hline 9 & 40 's & 22 & $M$ & Spec. & PCT & University \\
\hline 10 & 50 's & 30 & $\mathrm{~F}$ & Spec. & PCT & University \\
\hline II & 40 's & 23 & $M$ & Spec. & PCT & General \\
\hline 12 & 40 's & 20 & $F$ & Spec. & PCT & General \\
\hline 13 & 30 's & 11 & $M$ & Cert. & РCT & Cancer center \\
\hline 14 & 30 's & 13 & $M$ & Spec. & РСТ & University \\
\hline 15 & 40 's & 16 & $M$ & Spec./Onco & $\mathrm{PCT} / \mathrm{PCU}$ & Cancer center \\
\hline 16 & 50 's & 22 & $F$ & Spec. & РCT & Cancer center \\
\hline 17 & 40 's & 20 & $M$ & Spec. & РСТ & General \\
\hline 18 & 50 's & 30 & $M$ & Spec. & PCT/PCU & Cancer center \\
\hline 19 & 50 's & 30 & M & Spec. & РCT & General \\
\hline 20 & 50 's & 24 & $\mathrm{~F}$ & Spec. & PCT & University \\
\hline 21 & 30 's & 13 & $M$ & Spec. & PCT & General \\
\hline
\end{tabular}

Notes: ${ }^{2}$ Acquisition of breast specialization: Cert. (Board Certified member of The Japanese Breast Cancer Society), Spec. (Specialist of The Japanese Breast Cancer Society), Onco (Diplomate, Subspecialty Board of Medical Oncology, JSMO), ${ }^{b}$ Hospital Palliative Care Resource: PCT (Palliative Care Team), ${ }^{\text {CH}}$ Hospital: Cancer (Designated cancer hospital), University (University hospital), General (General hospital), Cancer center (National cancer center)

Abbreviation: PCU, Palliative Care Unit.

\section{Box I Set of interview questions}

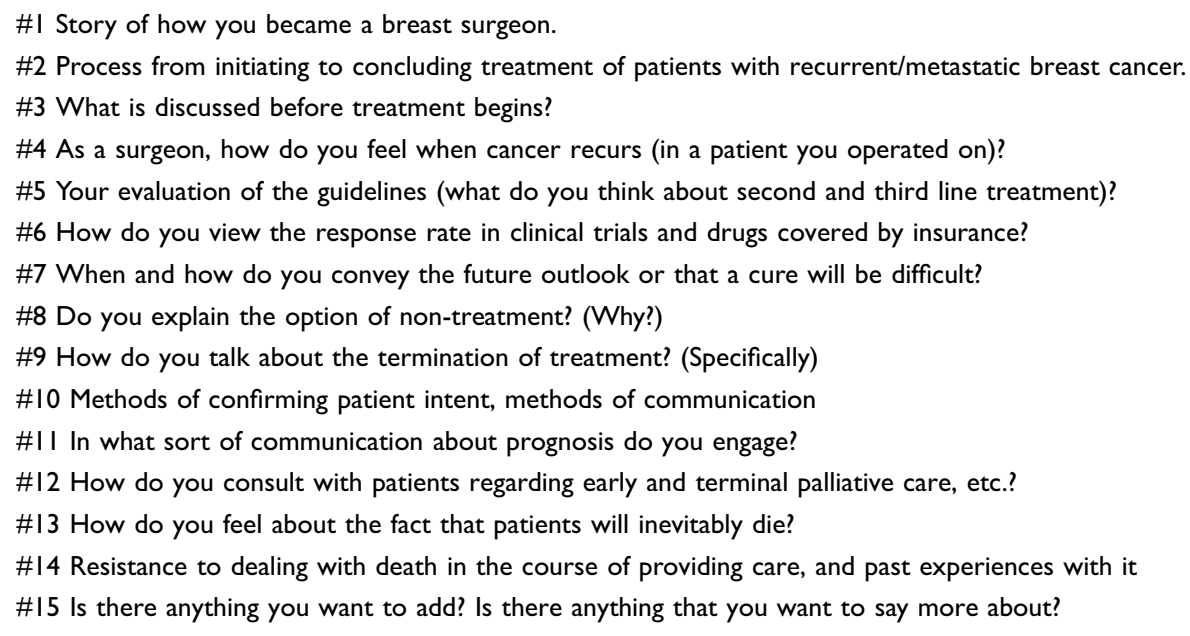

they ended it in order to avoid doing harm, although they kept patients still hoping for continued treatment. The other is a "belief that the patient is an entity who can accept death" (hereafter "acceptance belief"). Doctors with this belief provided patients with an overview of their conditions early on following the diagnosis of recurrent/metastatic breast cancer.
They repeatedly prepared patients for bad news about the progression of the illness or termination of treatment from early in the treatment process, and ultimately, when they judged that administering the next treatment would make it impossible for patients to achieve their own good death processes, they proposed terminating treatment. 


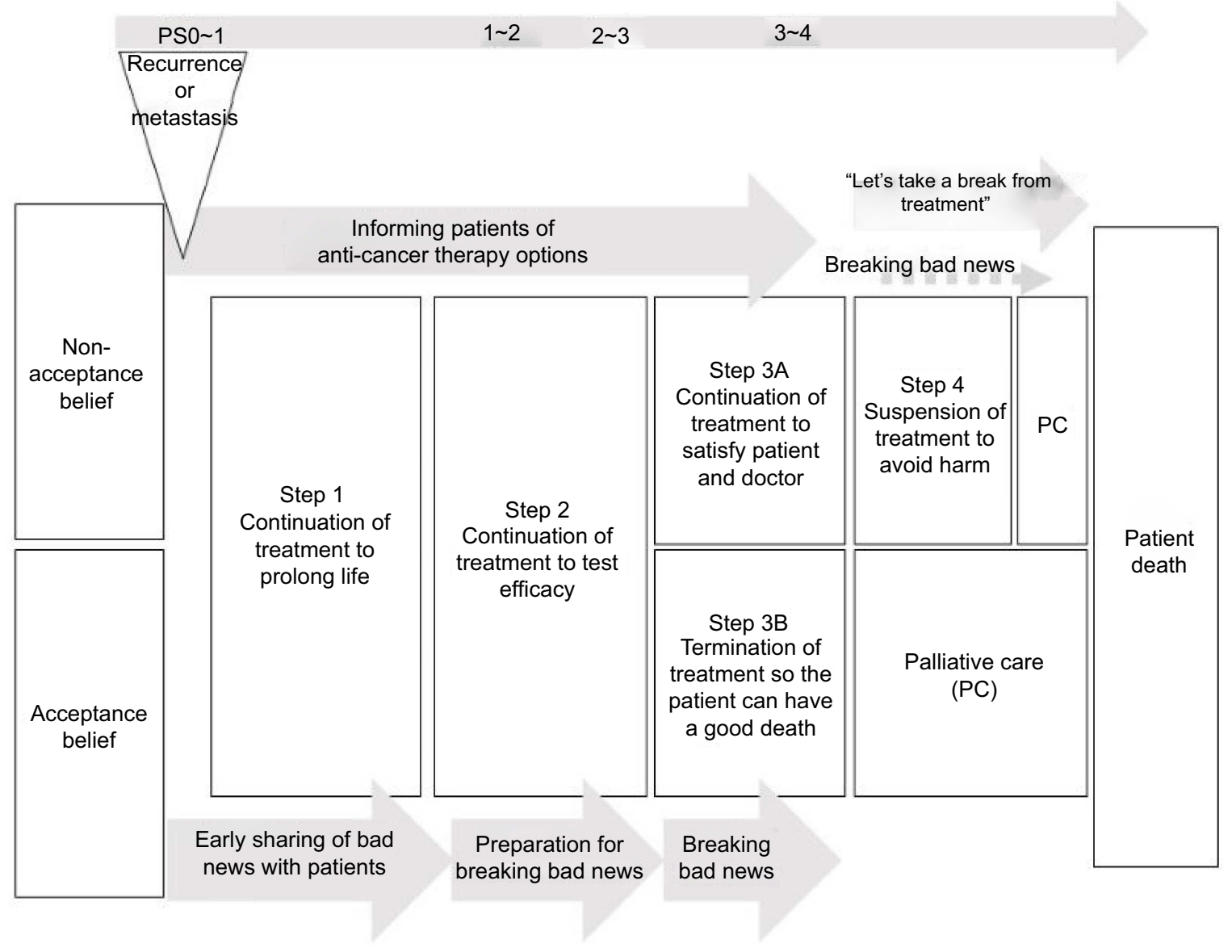

Figure I The process based on two beliefs.

\section{Non-acceptance belief narratives}

First, doctors with the non-acceptance belief considered patients unable to accept death, and avoided discussions with patients that involved confronting death.

For Japanese people, I think it's hard to end life in a way that simply accepts death. There are few who have the strength to face death, and even fewer doctors who are willing to accompany them as they do so. (ID8)

Many had a sense of powerlessness and guilt toward patients whose cancer relapsed after they performed surgery, and thus, as surgeons, were adamant about continuing chemotherapy. They stated that, as the person who understands patients the best, they wanted to continue to be in charge of their care until the very end. This kind of doctor believed that it was the surgeon's mission to continue to support the life of patients with treatment.

My feeling is that really the stance of the surgeon is to be proactive about surgery and to always think about how to do things better. (ID7)

\section{Acceptance belief narrative}

On the other hand, doctors with the acceptance belief considered patients to be able to accept death, and aspired for communication that would support patients as they face death. They believed that the relationship of trust with patients required a sharing of an overarching perspective on the disease that included death.

By giving patients some picture of what the illness will be like in advance, I think they can make their own judgments, or maybe by doing that, even when I have to give them bad news, they would be able to accept it. (ID15)

Furthermore, this group believed that the doctor's mission was to keep supporting patients so they could experience a good death. They believed it most important to ensure that patients are able to spend their final days as they wish.

It (the doctor's goal) is all about finding how we can support patients during the time they have left before they die. (ID12) 


\section{Four steps for decision-making process of chemotherapy for advanced breast cancer patients \\ Step I}

In Step 1, the doctor continues treatment to prolong life. When recurrence/metastasis is diagnosed, the first treatment option is chemotherapy, unless hormone therapy is indicated. Regardless of their beliefs, many doctors encouraged patients to undergo treatment, believing there is evidence that the initial treatment (first through third line treatment) prolongs life.

At this stage, doctors with the non-acceptance belief conveyed to patients that the illness will not get better, but many prioritized discussing specific anti-cancer therapies, rather than sharing an overview of the illness, which includes death.

When it recurs, I first discuss the necessary anti-cancer therapy. (ID14)

On the other hand, doctors with the acceptance belief shared with patients an overview of the illness that implies death will inevitably come, in order to build a relationship of trust with patients.

I make it a point to clearly tell them that if the cancer comes back, they will die eventually. Maybe I do this to avoid any misunderstanding. (ID12)

\section{Step2}

In Step 2, treatment is continued in order to test its potential efficacy. In the case of breast cancer patients, physical condition is often good even after failing the fourth line of treatment, and although the evidence of their efficacy is inadequate, multiple treatment options exist. Doctors, however, out of mistrust of current evidence or a belief that any drug approved and covered by public medical insurance must have at least some minimal effect, entrusted their hopes to any therapy that has a non-zero chance of working, and continued treatment.

It's not as if there's evidence that the treatment will never work. (ID15)

Oncologists stress the evidence, but breast surgeons want to provide patients with any treatment that would not do harm. There are also patients who want to try any treatment if it might have some effect. (ID 11)

At this point, doctors with the non-acceptance belief felt a resistance to discussing prognosis, and rarely shared the prognosis with patients. Doctors with the acceptance belief, however, were prepared to discuss all bad news, and at times shared an outlook on prognosis with patients.

If patients want to know how long they have left (prognosis), I feel I should tell them how long I think they have to live. (ID2)

\section{Step3A}

Step 3 comes into play after failing the fifth line of treatment, and doctors already predict that the next treatment would not be effective. At this step, doctors with the nonacceptance belief continued treatment in order to satisfy patients, hoping the treatment would have a positive psychological effect (Step 3A). At this stage, doctors stressed satisfying patients by continuing treatment, rather than the effect of the chemotherapy itself.

The point of treatment is to give peace of mind, for the patient's sake. (ID21)

Because doctors at this stage emphasized patient safety, they administered a reduced dosage when the patient's physical condition was bad, even if it weakened the anticancer effect. At this stage, continuing treatment is thought to have the psychological effect of offering salvation to maintain hope, or to help patients understand through experience that their condition is progressing and that treatment will no longer have an effect.

'We did all we could, but it didn't work; there's nothing more to do,' I want patients to come to that conclusion on their own. (ID4)

Furthermore, participants believed that fulfilling the wishes of patients would also satisfy the doctor. In the backdrop of this was the desire to live up to their ideal vision of the doctor as one who can fulfill patient hopes by always proactively proposing treatments. These doctors were confident that a surgeon could manage pain relief and end-of-life care. In addition, when continuing to diagnose and treat patients they had operated on, as surgeons, there was a sense of guilt at having allowed the cancer to return, and a feeling of powerlessness at being unable to heal it, leading them to insist on further chemotherapy. For this reason, they believed that the mission of doctors is to support the life of patients until the very end, motivating the continuation of treatment.

When cancer returns in patients I operated on, I feel guilty. That's why I want to do something (chemotherapy) for them. (ID18) 
Among these doctors, there was a tendency to avoid clearly confirming patient intent regarding treatment, out of a sense of resistance toward convincing the patient to give up treatment. Inferring the desire of patients to continue treatment, they suggest it as long as patients did not refuse, or alternatively did not recommend treatment but conveyed this in a way that left room for continuing treatment.

As long as they don't say they want to stop, we keep proposing options. (ID7)

Even when patients ask about prognosis, these doctors often did not clearly share it to avoid hurting them. Reasons for this included the difficulty of prognosis in breast cancer, or that it was not necessary to inform patients because they should have already realized, or that doctors hope the patients would guess the prognosis on their own during the course of treatment.

When continuing the treatment of metastasis or recurrence, I think patients probably know that it's natural that the treatment will stop working, and realizes that this is harsh, and risky. So, as a doctor, I think, ok, it's time to notice the signs. (ID9)

These doctors, however, sometimes looked back at the results of continued treatment at this stage and regretted the decisions made, wishing they had not gone through with the final treatment.

You wonder if it might have been better not to proceed with that last treatment. (ID4)

\section{Step3B}

On the other hand, doctors with the acceptance belief terminated treatment for the sake of allowing patients to have a good death (Step 3B). These doctors drew on information gleaned from discussions with patients until that point, and when it seemed highly likely that the next treatment would make it impossible for them to have a good death, they would propose giving up on treatment in favor of palliative medicine.

If we pursue treatment, there is the possibility that patients might not be able to do the things that are most precious to them, so I let them know that it's better not to do it (anticancer therapy). (ID16)

Many patients accepted such proposals, and anti-cancer therapy was terminated to focus solely on palliative medicine, so that the case does not progress to Step 4. When patients had difficulty accepting death, and strongly requested that treatment be continued, doctors respected these intentions and attempted treatment for a limited period. When treatment was found not to be effective, however, these doctors proposed terminating treatment, and almost all patients accepted this.

If someone really wants to continue, I say ok, let's do our best. But this is the last thing we'll do, I explain. I tell them that if this doesn't work, we quit. (ID15)

Some doctors with the acceptance belief had this kind of experience with patients: as a result of their attempts to persuade a patient to terminate anti-cancer therapy and focus on palliative care, the patient stops coming to the hospital and transfers to a different hospital in order to continue treatment.

(After even the patient mentioned wanting to proceed with treatment) The husband asked me to 'just go on and do it for her.' I explained that it was better to value the time remaining (rather than continuing treatment), but they said, 'Fine, we will go to another hospital,' and the patient died just one month after transferring. (ID17)

\section{Step4}

When the illness worsened to the point that chemotherapy would be dangerous, doctors with the non-acceptance belief decided they could not perform treatments that would only harm patients, and paused treatments in order to avoid harm (Step 4).

At this time, while doctors sometimes told patients they could not offer any more treatment, they did show a sense of consideration to protect patients from the harsh reality of facing death, and so framed their proposal as "Let's take a break from treatment for a bit." This allowed patients to maintain hope that treatment would be reinitiated, while effectively ending treatment.

When treatment was virtually impossible to continue, I would say something like 'Why not take a little break?' This is different than saying that no more treatment would be performed. (ID6)

\section{Discussion}

\section{Beliefs of doctors and the anti-cancer therapy process}

In this study, we identified two beliefs held by doctors who treat recurrent/metastatic breast cancer-the acceptance 
belief and non-acceptance belief - and created a model for the treatment process and communication shaped by differences in these beliefs. Pirl et al categorized the process of terminating chemotherapy in the terminal stage into (1) a final decision to clearly terminate treatment, (2) an ambiguous termination of treatment through postponement of the decision, and (3) a "non-decision" that leaves treatment terminated. ${ }^{10}$ Doctors of the present study who held the acceptance belief followed (1), whereas doctors with the non-acceptance belief almost always followed (2) or (3). The research by Pirl et al, however, merely classified patterns. The present study is unique in that it clarified the underlying unconscious thoughts of doctors.

\section{Overprotection model in patient-doctor relationships}

Among doctors who held the non-acceptance belief, there were those who, not wanting to hurt patients, inferred that the intent of patients was a "desire for treatment" and thus continued to propose treatment options. Doctors taking this approach consistently throughout the process of treating patients with recurrent/metastatic breast cancer may have hindered the psychological growth of patients because, if they had adequately discussed the issues during the process, patients may have come to accept and understand death. This resembles the type of overprotection that occurs when mothers obstruct their children's process of growth and autonomy in order not to harm them. In this study, we refer to this type of patient-doctor relationship as "the overprotection model".

One model of patient-doctor relations in which doctors make decisions against patient wishes or by inferring intent is paternalism, but "the overprotection model" is different. Paternalism can be divided into "hard paternalism" in which doctors know the patient's intent but go against it nonetheless, and "soft paternalism", when doctors do not know the patient's intent but they or patient family members make decisions thought to be best for patients. ${ }^{11}$ In the overprotection model we propose, doctors impede patient growth. Doctors make decisions based on their unconscious beliefs, as they do not believe the patient's ability to grow, and deny the possibility of growth through the process of facing a good death. In other words, the doctor's stance is not to deny the patient's intent, but to deny growth of the patient as a human, which differs from traditional paternalism.

If, under the overprotection model, the patient's idea of a good death is to die without facing death, and this matches what doctors infer is a good death, then this model could be beneficial. Some have noted, however, that there may be patients who, even though they display nothing but rejection or anger when initially diagnosed, will come to grow, achieve autonomy, and accept death through the process of interacting with their doctors. ${ }^{12,13}$ Although this study did not delve into the drawbacks of adopting the overprotection model, we believe they would be substantial. Future studies that examine the effects of the overprotection model and include patients and bereaved family members as participants are warranted.

\section{Clinical implications}

Table 2 shows the 10 categories identified in this study, which serve as clues as to which belief the doctors themselves hold when they approach treatment. Based on whether there were more As or Bs, we considered it possible to determine that a doctor's beliefs were closer to "acceptance" for A or "non-acceptance" for B (though there may of course be doctors who hold both beliefs). Below we describe notable points for each belief.

\section{For doctors closer to the non-acceptance belief Try to share patient values to the extent possible} Doctors with the non-acceptance belief tended to avoid talking to patients about death, because they thought it would

Table 2 Clues as to which belief the doctors themselves hold when they approach treatment beliefs

\begin{tabular}{|l|l|}
\hline Acceptance group & $\begin{array}{l}\text { - Often discusses not just treatment but bad news, outlook } \\
- \text { Makes an effort to prepare to talk about bad news } \\
- \text { Has experienced being told by patients that they want to discuss bad news early } \\
- \text { Has experienced sharing bad news with patients who were able to accept a good death } \\
- \text { Good collaboration with palliative medicine, palliative care team }\end{array}$ \\
\hline Non-acceptance group & $\begin{array}{l}\text { - Discussing bad news will worsen relationship with patients, or has worsened it } \\
\text { - Patients are happier if they can make it to the end of life without considering death } \\
\text { - Often end treatment by saying, "let's take a little break from treatment" } \\
\text { - Thinks palliative medicine alone will not prolong patient life } \\
\text { - Feels guilty about postoperative recurrence }\end{array}$ \\
\hline
\end{tabular}


cause suffering. It is possible that, for individual patients, these doctors were unable to acquire information about what the patient considered a "good death". Although it might be difficult to share accurately the patient's idea of what constitutes a good death, the sense that doctors understand their thoughts is a major source of support for patients. In recent years, Advance Care Planning (ACP) has broadened ways of not only sharing patient intentions about treatment, but even sharing their value systems. There have been reports that ACP has increased the satisfaction of terminal patients and their family members. ${ }^{14}$ Thus, the sharing of values, not only through ACP, could provide valuable support to patients.

\section{Coordinating treatment with the palliative care team}

Disclosing bad news on disease outlook, including death, is a difficult and stressful kind of communication for doctors. ${ }^{15-17}$ Particularly when a doctor is in charge of the care of a single patient over a long period, it can be very difficult for them to discuss the terminal stage. ${ }^{16}$ Temel et al reported that when discussing terminal stage care, an introduction to the palliative care team can be a valuable source of support. ${ }^{18}$ For doctors who resist discussing end of life, promptly informing patients about the palliative care team, and letting patients know about preparations to coordinate treatment with palliative care team, can potentially relieve doctors' stress around disclosing around bad news.

\section{For doctors closer to the acceptance belief}

Awareness of individual differences in "good death" desired by patients

Among doctors with the acceptance belief, there were a few whose values strongly insisted that only by accepting death could patients meet a good death, and thus they appeared not to address individual differences in what a good death might mean. In a previous qualitative study on patients with metastatic breast cancer, some patients were found to be overwhelmed by the fear of death, see treatment as a lifeline, and hope to continue treatment for as long as possible in order to forestall death. ${ }^{19}$ There is also a report that for some cancer patients, treatment is used as a form of life support and way of coping with the fear of death; when treatment is stopped, it eases the strain on the body, but leaves emotional stress. ${ }^{20}$ That study also suggested that continuing chemotherapy at a dosage that would not impact the patient physically might offer salvation to such patients. In cases where patients absolutely desire to continue treatment even after discussions, it may be prudent to examine how to respect the patient's conception of a good death.

\section{Limitations}

This study has some limitations. First, because we did not survey the views of patients or bereaved family members, we could not determine the impact of the treatment process or doctor's thinking on their QOL in the terminal stage. In this regard, surveys that include patients and family members and assessing their connection to the treatment process and doctor's way of thinking would be informative. Second, the present study is a qualitative study focused on Japanese breast surgeons, and the results are dependent on Japanese treatment practices and individual contexts. For this reason, gauging the generalizability of these results in other medical cultures and contexts will require an expanded scope, for example global quantitative research. By drawing from the rigorous procedures of the Grounded Theory Approach, however, this study has elucidated the target phenomenon in great detail.

\section{Conclusion}

This study identified two beliefs regarding the patient's acceptance of death among breast surgeons treating recurrent/metastatic cancer, and constructed a model of the terminal stage treatment process, consisting mainly of palliative chemotherapy and patient-doctor communication, based on relationships with these two beliefs. We propose that doctors who hold these beliefs, even partially, heed three points in the course of clinical practice: the need to share the patient's value system, the need to consider how best to make use of the palliative care team, and the need to be conscious of individual differences in what the patient regards as a good death. We believe this would contribute to the ability of doctors, who make decisions about chemotherapy at the end stage for breast cancer patients in the absence of clearly-defined guidelines, to offer patients optimal care.

\section{Acknowledgments}

The authors wish to thank the 21 breast surgeon informants who took part in this research for sharing their experiences and opinions. This work was supported by 'Support of Academic Researchers from The Uehiro Foundation on ethics and education'.

\section{Author contributions}

All authors contributed to data analysis, drafting and revising the article, gave final approval of the version to be published, and agree to be accountable for all aspects of the work. 


\section{Disclosure}

Dr Taichi Hatta reports grants from Supporting Program for InteRaction-based Initiative Team Studies (SPIRITS), Research Institute of Science and Technology for Society (RISTEX), outside the submitted work. The authors report no other conflicts of interest in this work.

\section{References}

1. Earle CC, Neville BA, Landrum MB, Ayanian JZ, Block SD, Weeks JC. Trends in the aggressiveness of cancer care near the end of life. J Clin Oncol. 2004;22(2):315-321. doi:10.1200/ JCO.2004.08.136

2. Lee HS, Chun KH, Moon D, Yeon HK, Lee S, Lee S. Trends in receiving chemotherapy for advanced cancer patients at the end of life. BMC Palliat Care. 2015;14:4. doi:10.1186/s12904-015$0001-7$

3. Liu TW, Chang WC, Wang HM, et al. Use of chemotherapy at the end of life among Taiwanese cancer decedents, 2001-2006. Acta Oncol. 2012;51(4):505-511. doi:10.3109/0284186X.2011.653440

4. Schildmann J, Tan J, Salloch S, Vollmann J. "Well, I think there is great variation ..." ": a qualitative study of oncologists' experiences and views regarding medical criteria and other factors relevant to treatment decisions in advanced cancer. Oncologist. 2013;18(1):90-96. doi:10.1634/ theoncologist.2012-0206

5. The AM, Hak T, Koeter G, van Der Wal G. Collusion in doctor-patient communication about imminent death: an ethnographic study. BMJ. 2000;321(7273):1376-1381. doi:10.1136/bmj.321.7274.1450

6. Laryionava K, Heussner P, Hiddemann W, Winkler EC. Framework for timing of the discussion about forgoing cancer-specific treatment based on a qualitative study with oncologists. Support Care Cancer. 2015;23(3):715-721. doi:10.1007/s00520-014-2416-8

7. Corbin JSA Basics of qualitative research (3rd ed.): techniques and procedures for developing grounded theory. In: Thousand Oaks, California; 2008. Available from: http://methods.sagepub.com/book/ basics-of-qualitative-research. Accessed December 18, 2018.
8. Lincoln YS, Guba YSLEG, Guba EG, Publishing S. Naturalistic Inquiry. Thaousand Oaks, CA: SAGE Publications; 1985.

9. Creswell JW. Research Design: Qualitative, Quantitative, and Mixed Methods Approaches. Thaousand Oaks, CA: SAGE Publications; 2003.

10. Pirl WF, Greer JA, Irwin K, et al. Processes of discontinuing chemotherapy for metastatic non-small-cell lung cancer at the end of life. J Oncol Pract. 2015;11(3):e405-412. doi:10.1200/JOP.2014.002428

11. Dworkin G, Sugden SJB. Paternalism. Monist. 1972;56(1):64-84. doi:10.5840/monist197256119

12. Lyckholm LJ. Thirty years later: an oncologist reflects on KublerRoss's work. Am J Bioethics. 2004;4(4):W29-31. doi:10.1080/ 15265160490908059

13. Zimmermann C. Acceptance of dying: a discourse analysis of palliative care literature. Soc Sci Med. 2012;75(1):217-224.

14. Detering KM, Hancock AD, Reade MC, Silvester W. The impact of advance care planning on end of life care in elderly patients: randomised controlled trial. BMJ. 2010;340:c1345. doi:10.1136/bmj.c293

15. Bernacki RE, Block SD. Communication about serious illness care goals: a review and synthesis of best practices. JAMA Intern Med. 2014;174(12):1994-2003. doi:10.1001/jamainternmed.2014.5271

16. Fallowfield L, Jenkins V. Communicating sad, bad, and difficult news in medicine. Lancet. 2004;363(9405):312-319. doi:10.1016/S01406736(03)15392-5

17. Studer RK, Danuser B, Gomez P. Physicians' psychophysiological stress reaction in medical communication of bad news: A critical literature review. Int J Psychophysiol. 2017;120:14-22. doi:10.1016/ j.ijpsycho.2017.06.006

18. Temel JS, Greer JA, Admane S, et al. Longitudinal perceptions of prognosis and goals of therapy in patients with metastatic non-small-cell lung cancer: results of a randomized study of early palliative care. J Clin Oncol. 2011;29(17):2319-2326. doi:10.1200/JCO.2010.32.4459

19. Bergqvist J, Strang P. The will to live - breast cancer patients perceptions' of palliative chemotherapy. Acta Oncol. 2017;56 (9):1168-1174. doi:10.1080/0284186X.2017.1327719

20. Buiting HM, Terpstra W, Dalhuisen F, et al. The facilitating role of chemotherapy in the palliative phase of cancer: qualitative interviews with advanced cancer patients. PLoS One. 2013;8(11):e77959. doi:10.1371/journal.pone.0077959
Breast Cancer - Targets and Therapy is an international, peer-reviewed open access journal focusing on breast cancer research, identification of therapeutic targets and the optimal use of preventative and integrated treatment interventions to achieve improved outcomes, enhanced survival and quality of life for the cancer patient.
The manuscript management system is completely online and includes a very quick and fair peer-review system, which is all easy to use. Visit http://www.dovepress.com/testimonials.php to read real quotes from published authors. 